\title{
Nanoscale Fluid Transport: Size and Rate Effects
}

\author{
Xi Chen, ${ }^{\dagger}$ Guoxin Cao, ${ }^{\dagger, \|}$ Aijie Han, ${ }^{\ddagger}$ Venkata K. Punyamurtula, ${ }^{\ddagger}$ Ling Liu,${ }^{\dagger}$

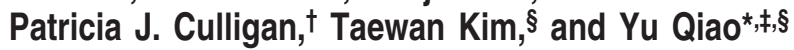

\begin{abstract}
Department of Civil Engineering and Engineering Mechanics, Columbia University, New York, New York 10027-6699, and Department of Structural Engineering, and

Program of Materials Science and Engineering, University of California-San Diego, La Jolla, California 92093-0085
\end{abstract}

Received July 10, 2008; Revised Manuscript Received August 5, 2008

\begin{abstract}
The transport behavior of water molecules inside a model carbon nanotube is investigated by using nonequilibrium molecular dynamcis (NMED) simulations. The shearing stress between the nanotube wall and the water molecules is identified as a key factor in determining the nanofluidic properties. Due to the effect of nanoscale confinement, the effective shearing stress is not only size sensitive but also strongly dependent on the fluid flow rate. Consequently, the nominal viscosity of the confined water decreases rapidly as the tube radius is reduced or when a faster flow rate is maintained. An infiltration experiment on a nanoporous carbon is performed to qualitatively validate these findings.
\end{abstract}

Introduction. Owing to their small length scales and large specific surface areas, nanoconduits play a key role in nanopipets, ${ }^{1}$ biomolecule separation, ${ }^{2}$ molecule detection, ${ }^{3}$ biocatalysis, ${ }^{4}$ encapsulation media for storage and transport, ${ }^{5}$ as well as energy absorption and damping. ${ }^{6-8} \mathrm{~A}$ critical challenge lies in understanding and controlling the unique transport behaviors of liquid molecules in confining nanoenvironments. Recent theoretical and experimental studies indicated that the flow rates of fluids in carbon nanotubes (CNTs) are much higher than the prediction of classic continuum theory. ${ }^{5,9-12}$ For example, Majumder et al. ${ }^{9}$ reported an experimental result that the liquid flow rates in nanotubes (with a diameter of about $7 \mathrm{~nm}$ ) were $4-5$ orders of magnitude faster than that at the macroscale, and, furthermore, the flow rate did not decrease with an increase in fluid viscosity. In molecular dynamics (MD) simulations, Hummer et al. ${ }^{11}$ and Skoulidas et al. ${ }^{10}$ also found that water or gas flew much faster inside a CNT than in a classical macroscale tube, suggesting that the effects of nanochannel size on intrinsic fluid properties, such as the viscosity, need to be reinvestigated at the nanoscale.

The results of investigations on nanoscale viscosity that are available in literature are sometimes contradictory to each other.

\footnotetext{
* Corresponding author. E-mail: yqiao@ucsd.edu.

$\dagger$ Columbia University.

${ }^{*}$ Department of Structural Engineering, University of California-San Diego.

$\S$ Program of Materials Science and Engineering, University of California-San Diego.

"Present address: Department of Engineering Mechanics, University of Nebraska at Lincoln, Lincoln, NE 68588.
}

Among prior experiments, Raviv et al. ${ }^{13}$ indicated that when water molecules were confined between two curved mica surfaces (the water film thickness, $h$, is $0.4-3.5 \mathrm{~nm}$ ), the effective viscosity, $\eta$, remained similar to its bulk counterpart. Conversely, when $\mathrm{Li}$ et al. ${ }^{14}$ studied water films with a thickness $h<2 \mathrm{~nm}$, they found that $\eta$ was higher than its bulk counterpart by 4 orders of magnitude on hydrophilic surfaces (mica, glass), whereas no size effect on hydrophobic surfaces (graphite) could be detected. In addition, Major et al. ${ }^{15}$ reported that $\eta$ of water increased over 7 orders of magnitude when $h$ was less than $1 \mathrm{~nm}$. Note that these experimental measurements can be quite sensitive to surface properties (e.g., defects and impurities), which may be one of the reasons why they were contradicting with each other. On the basis of atomistic simulations, Leng et al. ${ }^{16}$ found that the viscosity of a water film with $h=2.44 \mathrm{~nm}$ was close to that of bulk water, while when $h=0.92 \mathrm{~nm}$ the viscosity increased by 2 orders of magnitude. By using the Green-Kubo relation from the linear response theory and a MD-based flux autocorrelation function, Liu et al. ${ }^{17}$ argued that inside a $(16,16) \mathrm{CNT}$, the viscosity of water was the same as the bulk viscosity, but it nearly doubled in a $(8,8)$ tube. Such results obtained from equilibrium MD simulations contradict with the very high flow rate of water in CNTs observed in MD simulations ${ }^{5,11}$ and experiments. ${ }^{9,12}$ Indeed, equilibrium MD simulation may be difficult for nanofluids since there are very few molecules along the radial direction inside the nanotube, and it is hard to experimentally verify the viscosity values obtained at zero flow rate in experiments. 
Currently, while it is generally accepted that, at the nanoscale, the liquid viscosity can differ considerably from its bulk value, definitive numerical and experimental analyses, particularly on the rate effects which often accompany the size effects, are still lacking. This deficiency is hindering further understanding of the fundamental nanofluidic properties, in particular, the causes of the prominent size effects and possible rate effects

For tube flow, in the continuum theory, no-slip boundary condition is often assumed, where the flow velocity at the tube wall $\left.v\right|_{r=R_{0}}=0$, with $r$ indicating the radial direction and $R_{0}$ indicating the channel/tube radius. This assumption no longer holds true for nanofluidic flow where the liquid molecules slide against the solid lattice. To describe the nanofluidic behavior, a parameter of slip length can be defined as an extrapolation of the pore radius, such that at a hypothetical tube wall the nonslip boundary condition is met. For different liquids, the slip length was reported to be around $3.4 \sim 68 \mu \mathrm{m} .{ }^{9}$ The slip length, however, is not a physical quantity and is thus hard to be employed in a definitive analysis. As an alternative, it may be more straightforward to examine the interaction between a nanochannel and the liquid molecules and to explore how such interaction affects the implications of this interaction on the liquid's nominal viscosity. The understanding of such behaviors may also shed light on other nanofluidic characteristics, such as water freezing in nanotubes..$^{18,19}$

Unique to a nanoenvironment is that the interaction between the solid wall and the liquid molecules accounts for most of the "resistance" that a steady flow would encounter, in contrast to the conventional viscosity force in a laminar flow (which accounts for the inherent dissipation in the interior). Because of the variation in the degree of confinement, such solid-liquid interactions can be size and rate dependent. It is therefore arguable that the shearing stress between the solid and liquid atoms, $\left.\tau\right|_{r=R}$, is the key characteristic of fluid transport at the nanoscale. Note that the area accessible to the liquid molecules is smaller than that enclosed by the solid wall, primarily because of the van der Waals (vdW) interaction, and thus the effective radius of the liquid segment, $R$, can be defined as $\left(R_{0}+R_{1}\right) / 2$, where $R_{1}$ is the diameter relevant to the first solvation shell liquid molecules (see Figure 1). The shearing stress $\left.\tau\right|_{r=R}$ will strongly affect the nominal liquid viscosity and fluid flow rate at the nanoscale, which is the main focus of the current study.

Molecular Dynamics Simulations. In order to explore the intrinsic behavior of nanoscale fluid transport, we carry out nonequilibrium molecular dynamics (NEMD) simulations (using LAMMPS ${ }^{20}$ ) on a model system and focus on the changes in $\left.\tau\right|_{r=R}$ as the nanochannel size and the fluid flow rate vary. The system includes water molecules inside a single-walled CNT with a periodical boundary condition imposed along the axial direction (Figure 1), and the TIP3P model is used for water molecules. ${ }^{21}$ The length of the computational cell is $300 \AA$. We have verified that the computational results are insensitive to the variation of the size of the cell. CNTs with sizes ranging from $(10,10)$ to



Figure 1. (a) Schematic of the initial structure and transport of water molecules in a model CNT. (b) The radial density profile (RDP) of water molecules inside CNTs with different radii. (c) The representative radial velocity profile (RVP) of water molecules inside a $(60,60)$ nanotube. Both RDP and RVP are computed via the average of all water molecules in the computational cell.

$(60,60)$ are analyzed, with $R_{0}$ varying from 0.675 to 4.053 $\mathrm{nm}$. The average flow speed (or flow rate), $\bar{v}$, is related to the flux $Q$ as $\bar{v}=Q /\left(\pi R^{2}\right)$. Although tube flexibility may affect transport behaviors, ${ }^{22}$ in order to reveal the intrinsic nanofluidic motions, we assume that the tube is rigid and such assumption is also consistent with the parallel experiments described below. The number of the water molecules inside the nanotube $(N)$ is chosen such that the initial water density is $0.998 \mathrm{Mg} / \mathrm{m}^{3}$ inside the occupied volume. The cutoff distance for the van der Waals interactions and the real-space part of the Coulomb interactions are set to be 10 $\AA$; the long-range Coulomb interactions are handled by using the particle-particle particle-mesh technique ${ }^{23}$ with a rootmean-square accuracy of $10^{-4}$. The time step in simulation is $1 \mathrm{fs}$. The initial structures are optimized for $100 \mathrm{ps}$ by using NVT ensemble, and the temperature is kept at $298 \mathrm{~K}$ by using Nose-Hoover thermostat.

After initial optimization, a constant acceleration is applied to each atom to create a uniform flow inside the tube, and after the desired flux (or $\bar{v}$ ) is reached, the force is removed. The NVE ensemble is used to monitor the velocity of water molecules in the next $100 \mathrm{ps}$, and during each time step, the instantaneous average flow speed of all molecules $(\tilde{v})$ is obtained (which decrease almost linearly with time). From the deceleration of the water flow $(a)$, the effective shearing stress between the tube wall and the water molecules is calculated as $\tau=\mathrm{Nma} /(2 \pi R L)$ (which is a function of both the tube size $R_{0}$ and the flow speed $\bar{v}$ ), where $m$ is the mass 


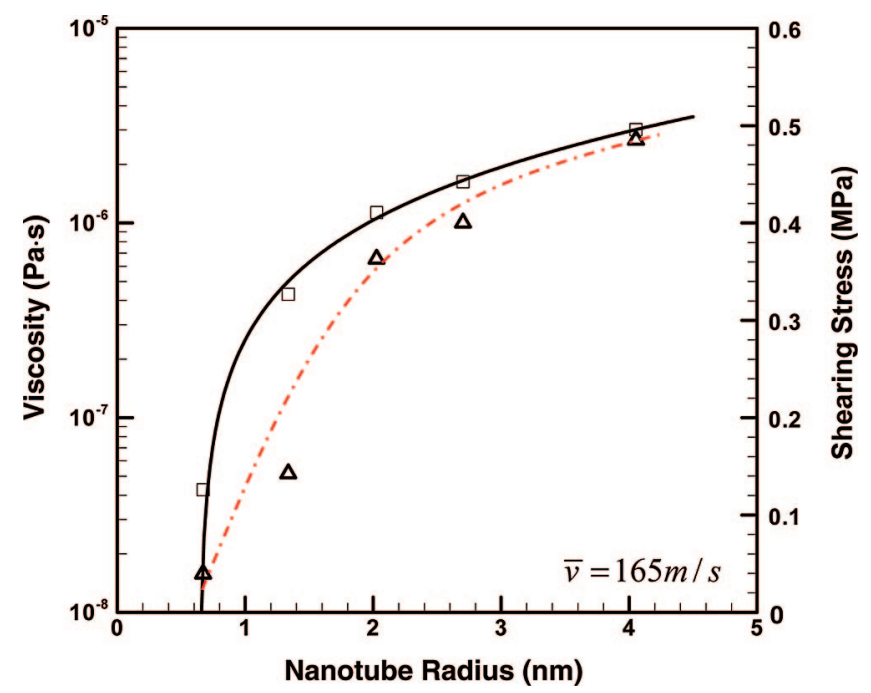

Figure 2. Size effect of shearing stress (triangle) and nominal viscosity (square), with $\bar{v}=165 \mathrm{~m} / \mathrm{s}$.

of a water molecule and $L$ is the tube length. Denoting $n_{0}$ as the number density of water, we can calculate the shearing stress as $\left.\tau\right|_{r=R} \equiv \tau=n_{0} m R a / 2$, which is a function of both the tube size and the average flow speed.

Figure 2 shows the relationship between $\tau$ and $R_{0}$ for different tube radii, in which $\bar{v}$ is fixed at about $165 \mathrm{~m} / \mathrm{s}$. The shearing stress $\tau$ increases with $R_{0}$, and the rate of increase is smaller for larger tubes. Overall, $\tau$ is relatively small in the tube size range under investigation, which shows that the CNT surface is quite smooth and the water molecules can easily slip against it. In fact, the shearing stress is primarily caused by the vdW interactions between the solid wall and the water molecules in the first solvation shell. The characteristic distance between the first solvation shell and the tube wall $(d)$ depends on the equilibrium distance between $\mathrm{O}$ and $\mathrm{C}$ atoms, the distribution of solid wall atoms, and the tube curvature. As a first order approximation, the magnitude of the first solvation shell scales with $1-d_{0} / R_{0}$, where $d_{0}=$ $d l_{R_{0} \rightarrow \infty}^{\bar{v}=0}$. This trend qualitatively agrees with the computed radial density profile (RDP) of water molecules inside the nanotubes (Figure 1). The magnitude of the first solvation shell increases with $R_{0}$, suggesting that a larger tube wall provides a more pronounced $\mathrm{vdW}$ constraint on the water molecules, and thus $\tau$ follows the same trend.

One interesting phenomenon is that the shearing stress is also dependent on the flow rate (Figure 3). In the example of a $(20,20)$ tube, $\tau$ increases with $\bar{v}$, with the rate of increase slowing down for higher values of $\bar{v}$. (This phenomenon is consistent with our recent study ${ }^{24}$ on the pressure-induced infiltration of water molecules into a nanochannel, showing that, in order to continuously overcome the energy barriers among the tetrahedral sites, a higher external pressure is required to sustain water infiltration into a nanochannel as the loading rate increases.)

With a high $\bar{v}$, as the water molecules move along the tube surface, there is no sufficient time for the liquid molecules to fully adjust their positions to minimize the system free energy, and the distance between the $\mathrm{C}$ atoms

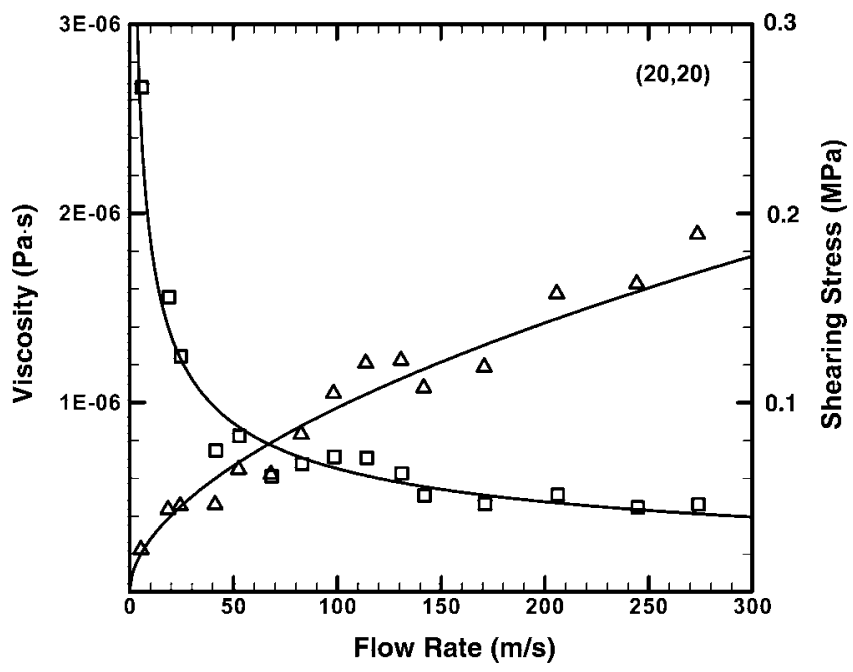

Figure 3. Flow rate effect of shearing stress (triangle) and nominal viscosity (square), with $R=1.336 \mathrm{~nm}$.

and adjacent water molecules can be less than the vdW equilibrium distance, which increases the overall $\mathrm{vdW}$ repulsion and leads to a higher shearing stress. ${ }^{24}$ Consider a water molecule that has to continuously overcome the energy barrier per unit length, $U$, whose derivative is proportional to $\tau$. For a first order assessment, $U$ can be stated as a power law function of the distance between the water molecule's current position and its vdW equilibrium position, which scales with the flow rate, as discussed above. Therefore, $\tau \sim \bar{v}^{m}$ and, according to Figure $3, m \approx 0.5$ for the present CNT analyzed in the current MD simulation.

The variation in the shearing stress directly causes the dependence of the effective liquid viscosity on the tube size and the flow rate. For a representative $\bar{v}=165 \mathrm{~m} / \mathrm{s}$, Figure 1 shows the radial velocity profile (RVP) of water molecules inside a $(60,60)$ nanotube, which is of a typical plug-flow profile, consistent with the work reported in ref 25. According to classic fluid mechanics, with a pressure difference of $\Delta P$, along a tube of length $L$, the flux is $Q=\pi R^{4} \Delta P /(8 \eta L)$. Since $\tau=\Delta P R /(2 L)$, the nominal viscosity of the transporting liquid can be assessed as $\eta=\tau \cdot R /(4 \bar{v})$. Note that, although this equation is based on continuum theory, it can be extended to complex flow ${ }^{26,27}$ to obtain the nominal viscosity at nanoscale (for the purpose of self-comparison; the most dominant nanofluidic characteristic is still the shearing stress discussed above). Figure 3 shows the relationship between $\eta$ and $\bar{v}$ inside a $(20,20)$ tube: $\eta$ sharply reduces with the increase of flow rate, and it starts to converge when $\bar{v}>$ $150 \mathrm{~m} / \mathrm{s}$. For the current tube size and flow rate ranges, $\eta \sim 1 / \bar{v}^{0.5}$; such a trend arises from the $\tau-\bar{v}$ relationship shown in the same figure.

According to Figure 3 , at a high $\bar{v}$ the rate effect is negligible. Under this condition, the tube size effect on the nominal viscosity can be further analyzed. Figure 2 shows the relationship between the nanotube radius and the nominal viscosity $(\bar{v} \approx 165 \mathrm{~m} / \mathrm{s})$. Since $\tau$ increases with $R, \eta$ increases quickly in larger tubes by nearly 2 orders of magnitude, as $R_{0}$ is changed from 0.67 to $4.05 \mathrm{~nm}$. The nominal viscosity of water inside the $(10,10)$ tube is $8.5 \times 10^{-8} \mathrm{~Pa} \cdot \mathrm{s}$, which 


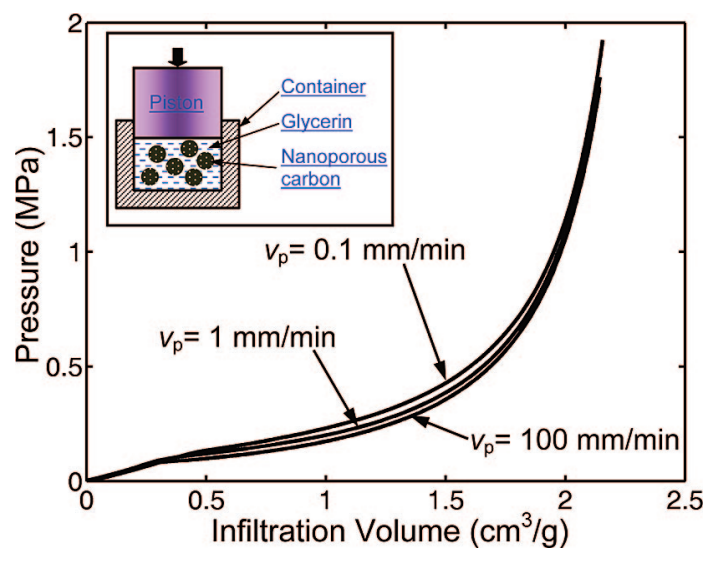

Figure 4. Typical sorption isotherm curves of the glycerin infiltration into the nanoporous carbon. The insert at the upper-left corner depicts the experimental setup.

is about 4 orders of magnitude lower than the viscosity of bulk water. This result is consistent with previous experimental and simulation results, ${ }^{5,9-12}$ indicating that the water flow rate at the nanoscale can be much higher than that of continuum fluids.

Experimental Section. To qualitatively validate the simulation results, we performed a pressure-induced infiltration experiment on a nanoporous carbon (Horn BP2000), whose pore surface is hydrophobic. ${ }^{28}$ The as-received material was in powder form, with the average particle size of $D$ $=10 \mu \mathrm{m}$. According to a gas absorption analysis, the specific nanopore volume was $V_{\text {pore }}=2.14 \mathrm{~cm}^{3} / \mathrm{g}$ and the specific nanopore surface area was $1175 \mathrm{~m}^{2} / \mathrm{g}$. The surface of the material was modified by using chlorotrimethylsilane. In a round-bottom flask, nanoporour carbon powders that had been vaccuum-dried for $12 \mathrm{~h}$ at $80^{\circ} \mathrm{C}$ were sealed in $40 \mathrm{~mL}$ of dry toluene. After $2.5 \%$ of chlorotrimethylsilane was injected into the flask, the mixture was refluxed at $90^{\circ} \mathrm{C}$ for $24 \mathrm{~h}$. Then, the carbon sample was filtered, rinsed repeatedly in dry toluene and acetone, and finally dried in vacuum at $50{ }^{\circ} \mathrm{C}$ for $12 \mathrm{~h}$.

The testing sample was prepared by immersing $0.3 \mathrm{~g}$ of the surface treated nanoporous carbon in $4 \mathrm{~g}$ of glycerin. Glycerin was employed as the liquid phase since its viscosity was much higher than that of water, so that the influences of nanopore structure and loading conditions could be amplified. The glycerin suspension was sealed in a steel cylinder by a steel piston with a gasket. By intruding the piston into the cylinder, an external pressure was applied on the liquid phase. The cross-sectional area of the piston, $A_{\mathrm{p}}$, was $286 \mathrm{~mm}^{2}$. The loading rate, $v_{\mathrm{p}}$, ranged from $0.1 \mathrm{~mm} /$ $\min$ to $100 \mathrm{~mm} / \mathrm{min}$. The pressure acting on the infiltrating liquid, $P$, was calculated as $F_{\mathrm{p}} / A_{\mathrm{p}}$, where $F_{\mathrm{p}}$ was the piston force. The infiltration volume was taken as $d_{\mathrm{p}} \cdot A_{\mathrm{p}}$, where $d_{\mathrm{p}}$ is the piston displacement. Typical sorption isotherm curves are shown in Figure 4. It can be seen that, as the external pressure increases, the system volume decreases rapidly in a certain pressure range, leading to the formation of an infiltration plateau. When $P$ is relatively low, the capillary effect of the nanopore walls cannot be overcome, and thus



Figure 5. Effective viscosity as a function of the nanopore radius and the loading rate.

the liquid does not enter the nanopores. With the increase of the pressure, the pressure-induced infiltration begins, and once the nanopores are filled, the system compressibility becomes small again. Because of the nanopore size distribution, the liquid infiltration starts with the largest nanopores, and the smaller nanopores can be involved in the infiltration process only when the pressure is sufficiently high. The nominal flux in a nanopore with a radius of $R$ can be estimated as $Q=v_{\mathrm{p}} \cdot A_{\mathrm{p}} / N_{\mathrm{p}}$, where $N_{\mathrm{p}}$ is the nanopore number density. The value of $N_{\mathrm{p}}$ can be stated as $f \cdot V_{\text {pore }} / \pi R^{2} L$, with $L=D / 2$ being the effective infiltration depth and $f$ being the probability density of pore volume distribution, measured by the gas absorption analysis. ${ }^{25}$ According to the equation of $\eta=\tau \cdot R /(4 \bar{v})$, the nominal viscosity can be calculated as $\eta=(\pi / 8)\left(P R^{4} / Q L\right)$, where the shearing stress is $\tau=$ $P R /(2 L)$. The results are shown in Figure 5. It can be seen clearly that the size and rate dependence of $\eta$ agrees qualitatively with the results of the MD simulation. In all of the cases under investigation, the value of $\eta$ is much smaller than its bulk counterpart. When the nanopore radius changes from nearly $1 \mathrm{~nm}$ to about $10 \mathrm{~nm}$, the effective viscosity rises by 1 order of magnitude. A more significant change takes place as the loading rate increases from $0.1 \mathrm{~mm} / \mathrm{min}$ to $100 \mathrm{~mm} / \mathrm{min}$, which causes a variation of $\eta$ by 3-4 orders of magnitude. The mismatch between the simulation and the testing data may be related to the differences in nanoporous structure and liquid phase.

Conclusion. In summary, the transport behavior of water molecules inside a model carbon nanotube is investigated by using NEMD. The result shows that the nominal viscosity of the confined liquid not only is a function of the tube size, but also is strongly dependent on the flow rate. A detailed analysis of the simulation data indicates that the size effect and the rate effect are directly related to the shearing stress between the liquid molecules and the solid atoms, which increases with the tube radius and/or the flow rate. These findings are verified qualitatively by a pressure-induced infiltration experiment on a nanoporous carbon in glycerin.

Acknowledgment. The work was supported by ARO under Grant W911NF-05-1-0288, by NSF and Sandia National Laboratory under Grant CMMI-0623973, and by NSF under Grant CMMI-0643726. 


\section{References}

(1) Hong, M.-H.; Kim, K. H.; Bae, J.; Jhe, W. Appl. Phys. Lett. 2000, 77, 2604-2606.

(2) Jirage, K. B.; Hulteen, J. C.; Martin, C. R. Science 1997, 278, 655658.

(3) Deamer, D. W.; Akeson, M. Trends Biotechnol. 2000, 18, 147-151.

(4) Mitchell, D. T.; Lee, S. B.; Trofin, L.; Li, N.; Nevanen, T. K.; Söderlund, H.; Martin, C. R. J. Am. Chem. Soc. 2002, 124, 1186411865.

(5) Kalra, A.; Garde, S.; Hummer, G. Proc. Natl. Acad. Sci. 2003, 100, 10175-10180.

(6) Chen, X.; Surani, F. B.; Kong, X.; Punyamurtula, V. K.; Qiao, Y. Appl. Phys. Lett. 2006, 89, 241918.

(7) Liu, L.; Qiao, Y.; Chen, X. Appl. Phys. Lett. 2008, 92, 101927.

(8) Qiao, Y.; Cao, G.; Chen, X. J. Am. Chem. Soc. 2007, 129, 23552359.

(9) Majumder, M.; Chopra, N.; Andrews, R.; Hinds, B. J. Nature 2005, $438,44-44$.

(10) Skoulidas, A. I.; Ackerman, D. M.; Johnson, J. K.; Sholl, D. S. Phys. Rev. Lett. 2002, 89, 185901.

(11) Hummer, G.; Rasaiah, J. C.; Noworyta, J. P. Nature 2001, 414, 188190.

(12) Holt, J. K.; Park, H. G.; Wang, Y.; Stadermann, M.; Artyukhin, A. B.; Grigoropoulos, C. P.; Noy, A.; Bakajin, O. Science 2006, 312, 1034 1037.

(13) Raviv, U.; Laurat, P.; Klein, J. Nature 2001, 413, 51-54.
(14) Li, T.-D.; Gao, J.; Szoszkiewicz, R.; Landman, U.; Riedo, E. Phys. Rev. B 2007, 75, 115415.

(15) Major, R. C.; Houston, J. E.; McGrath, M. J.; Siepmann, J. I.; Zhu, X.-Y. Phys. Rev. Lett. 2006, 96, 177803.

(16) Leng, Y.; Cummings, P. T. Phys. Rev. Lett. 2005, 94, 026101.

(17) Liu, Y.; Wang, Q.; Wu, T.; Zhang, L. J. Chem. Phys. 2005, 123 (23), 234701.

(18) Koga, K.; Gao, G. T.; Tanaka, H.; Zeng, X. C. Physica A 2002, 314, $462-469$.

(19) Koga, K.; Zeng, X. C.; Tanaka, H. Phys. Rev. Lett. 1997, 79, 52625265.

(20) Plimpton, S. J. J. Comput. Phys. 1995, 117, 1-19.

(21) Jorgensen, W. L.; Chandrasekhar, J.; Madura, J. D.; Impey, R. W.; Klein, M. L. J. Chem. Phys. 1983, 79, 926-935.

(22) Chen, H.; Johnson, J. K.; Sholl, D. S. J. Phys. Chem. B 2006, 110, 1971-1975.

(23) Hockney, R. W.; Eastwood, J. W., Computer Simulation Using Particles. Adam Hilger-IOP: Bristol, U.K., 1988.

(24) Cao, G.; Qiao, Y.; Zhou, Q.; Chen, X. Molecular Simulation 2008, 88, 371-378.

(25) Hanasaki, I.; Nakatani, A. J. Chem. Phys. 2006, 124, 144708.

(26) Rothstein, J. P.; McKinley, G. H. J. Non-Newtonian Fluid Mech. 1999, $86,61-88$.

(27) Semwogerere, D.; Morris, J. F.; Weeks, E. R. J. Fluid Mech. 2007, 581, 437-451.

(28) Wang, Y.-G.; Egashira, M.; Ishida, S.; Korai, Y. Carbon 1998, 307, 314.

NL802046B 